\title{
Measurement of $\Gamma_{\mathrm{ee}}(\mathrm{J} / \psi)$ with KEDR detector
}

V.V. Anashin, ${ }^{a}$ V.M. Aulchenko, ${ }^{a, b}$ E.M. Baldin, ${ }^{a, b}$ A.K. Barladyan, ${ }^{a}$

A.Y. Barnyakov, ${ }^{a, b}$ M.Y. Barnyakov, ${ }^{a, b}$ S.E. Baru, ${ }^{a, b}$ I.V. Bedny, ${ }^{a}$ A.E. Blinov, ${ }^{a, b}$

V.E. Blinov, ${ }^{a, b, c}$ A.V. Bobrov, ${ }^{a, b}$ V.S. Bobrovnikov, ${ }^{a, b}$ A.V. Bogomyagkov, ${ }^{a, b}$

A.E. Bondar, ${ }^{a, b}$ D.V. Bondarev, ${ }^{a}$ A.R. Buzykaev, ${ }^{a, b}$ S.I. Eidelman, ${ }^{a, b}$

Y.M. Glukhovchenko, ${ }^{a}$ V.V. Gulevich, ${ }^{a}$ D.V. Gusev, ${ }^{a}$ S.E. Karnaev, ${ }^{a}$ G.V. Karpov, ${ }^{a}$ S.V. Karpov, ${ }^{a}$ T.A. Kharlamova, ${ }^{a, b, 1}$ V.A. Kiselev, ${ }^{a}$ S.A. Kononov,,$^{a, b}$ K.Y. Kotov, ${ }^{a}$ E.A. Kravchenko, ${ }^{a, b}$ V.F. Kulikov, ${ }^{a, b}$ G.Y. Kurkin, ${ }^{a, c}$ E.A. Kuper, ${ }^{a, b}$ E.B. Levichev, ${ }^{a, c}$ D.A. Maksimov, ${ }^{a, b}$ V.M. Malyshev, ${ }^{a}$ A.L. Maslennikov, ${ }^{a, b}$ A.S. Medvedko, ${ }^{a}$

O.I. Meshkov,,$^{a, b}$ S.I. Mishnev, ${ }^{a}$ I.I. Morozov, ${ }^{a, b}$ N.Y. Muchnoi, ${ }^{a, b}$ V.V. Neufeld, ${ }^{a}$ S.A. Nikitin, ${ }^{a}$ I.B. Nikolaev, ${ }^{a, b}$ I.N. Okunev, ${ }^{a}$ A.P. Onuchin,,$^{a, b, c}$ S.B. Oreshkin, ${ }^{a}$ I.O. Orlov, ${ }^{a}$ A.A. Osipov,,$^{a, b}$ S.V. Peleganchuk,,$^{a, b}$ S.G. Pivovarov, ${ }^{a, c}$ P.A. Piminov, ${ }^{a}$ V.V. Petrov, ${ }^{a}$ A.O. Poluektov, ${ }^{a}$ I.N. Popkov, ${ }^{a}$ V.G. Prisekin, ${ }^{a, b}$ O.L. Rezanova, ${ }^{a, b}$ A.A. Ruban, ${ }^{a, b}$ V.K. Sandyrev, ${ }^{a}$ G.A. Savinov, ${ }^{a}$ A.G. Shamov, ${ }^{a, b}$ D.N. Shatilov, ${ }^{a}$ B.A. Shwartz, ${ }^{a, b}$ E.A. Simonov, ${ }^{a}$ S.V. Sinyatkin, ${ }^{a}$ Y.I. Skovpen, ${ }^{a}$ A.N. Skrinsky, ${ }^{a}$ V.V. Smaluk, ${ }^{a}$ A.V. Sokolov, ${ }^{a, b}$ A.M. Sukharev, ${ }^{a, b}$ E.V. Starostina, ${ }^{a, b}$ A.A. Talyshev, ${ }^{a, b}$ V.A. Tayursky, ${ }^{a, b}$ V.I. Telnov, ${ }^{a, b}$ Y.A. Tikhonov, ${ }^{a, b}$ K.Y. Todyshev, ${ }^{a, b}$ G.M. Tumaikin, ${ }^{a}$ Y.V. Usov, ${ }^{a}$ A.I. Vorobiov, ${ }^{a}$ A.N. Yushkov, ${ }^{a}$ V.N. Zhilich, ${ }^{a, b}$ V.V. Zhulanov ${ }^{a, b}$ and A.N. Zhuravlev ${ }^{a, b}$

\footnotetext{
${ }^{a}$ Budker Institute of Nuclear Physics,

11, akademika Lavrentieva prospect, Novosibirsk, 630090 Russia

${ }^{b}$ Novosibirsk State University,

2, Pirogova street, Novosibirsk, 630090 Russia

${ }^{c}$ Novosibirsk State Technical University,

20, Karl Marx prospect, Novosibirsk, 630092 Russia

E-mail: T.A.Kharlamova@inp.nsk.su
}

${ }^{1}$ Corresponding author. 
Abstract: The product of the electronic width of the $J / \psi$ meson and the branching fractions of its decay to hadrons and electrons has been measured using the KEDR detector at the VEPP-4M $e^{+} e^{-}$collider. The obtained values are

$$
\begin{aligned}
\Gamma_{e e}(J / \psi) & =5.550 \pm 0.056 \pm 0.089 \mathrm{keV}, \\
\Gamma_{e e}(J / \psi) \cdot \mathcal{B}_{\text {hadrons }}(J / \psi) & =4.884 \pm 0.048 \pm 0.078 \mathrm{keV}, \\
\Gamma_{e e}(J / \psi) \cdot \mathcal{B}_{e e}(J / \psi) & =0.3331 \pm 0.0066 \pm 0.0040 \mathrm{keV} .
\end{aligned}
$$

The uncertainties shown are statistical and systematic, respectively. Using the result presented and the world-average value of the electronic branching fraction, one obtains the total width of the $J / \psi$ meson:

$$
\Gamma=92.94 \pm 1.83 \mathrm{keV}
$$

These results are consistent with the previous experiments.

KEYWORDS: e+-e- Experiments

ARXIV EPRINT: 1801.01958 


\section{Contents}

1 Introduction 1

2 VEPP-4M collider and KEDR detector 2

3 Experiment and data sample $\quad 3$

$4 e^{+} e^{-}$cross section in vicinity of a narrow resonance 4

5 Data analysis $\quad \mathbf{5}$

5.1 MC simulation 5

5.2 Trigger requirements 6

$\begin{array}{lll}5.3 & \text { Luminosity determination } & 6\end{array}$

5.4 Selection of hadronic events 8

5.5 Fitting of the data 8

6 Study of systematic uncertainties 10

$\begin{array}{lll}6.1 & \text { Luminosity uncertainties } & 11\end{array}$

6.2 Uncertainty due to imperfect simulation of $J / \psi$ decays 12

$\begin{array}{lll}6.3 & \text { Detector-related uncertainties } & 15\end{array}$

$\begin{array}{ll}6.4 \text { Accelerator uncertainties } & 16\end{array}$

$\begin{array}{lll}6.5 & \text { Other uncertainties } & 17\end{array}$

$\begin{array}{lll}7 & \text { Summary } & 17\end{array}$

\section{Introduction}

The $J / \psi$ resonance, a bound state of $c \bar{c}$ quarks, was discovered more than forty years ago but its investigation is still actual. Fundamental properties of this meson including the branching fractions of leptonic and hadronic decays are important for understanding the quarkonium decay dynamics. The leptonic width of the $J / \psi$ meson is used in calculations of c-quark mass [1,2] and the hadronic contribution to the muon $g-2[3]$. It is also used for various calculations of radiative corrections due to the vacuum polarization and the initial-state radiation. The current precision of $\Gamma_{e e}$ in the potential models and in the lattice QCD (LQCD) calculations $[4,5]$ is compatible with that of the world-average value [6] and increase of the experimental precision for this value can be crucial for further development of the LQCD calculation techniques.

Measurements of the $J / \psi$ widths have a long history. They were studied at MarkI [7] and ADONE [8], and later at BES [9], BaBar [10], CLEO [11], KEDR [12, 13] and BESIII [14]. Usually $\Gamma_{e e}$ is measured in $J / \psi$ decays to hadrons, $e^{+} e^{-}$or $\mu^{+} \mu^{-}$final states 


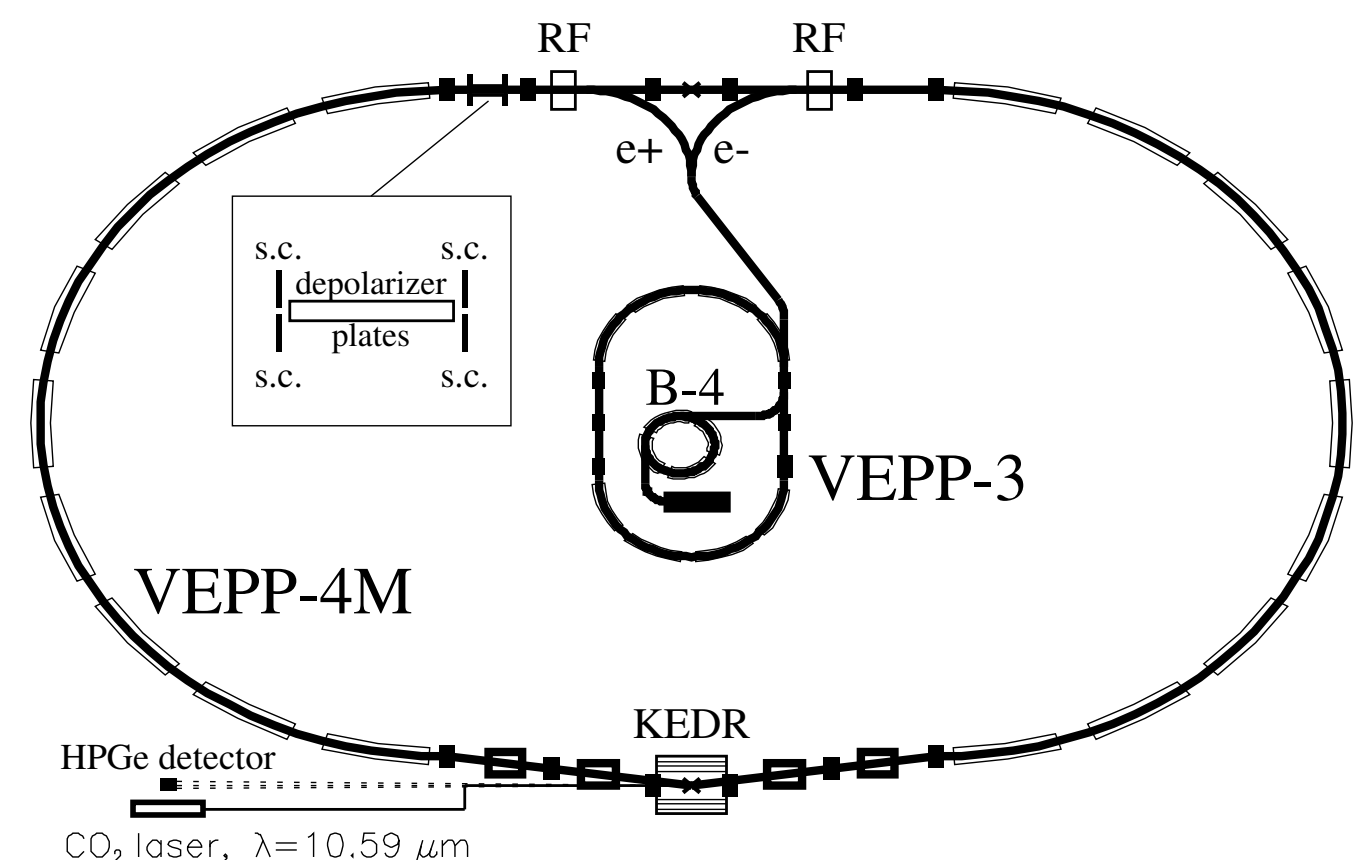

Figure 1. The VEPP-4M $e^{+} e^{-}$collider with KEDR detector.

and the obtained value is the product of $\Gamma_{e e}$ to the corresponding branching fraction. At present the best accuracy in the determination of $\Gamma_{e e}$ has been obtained by the BESIII collaboration [14] based on the $\Gamma_{e e} \cdot \mathcal{B}_{\mu \mu}(J / \psi)$ measurement in the initial-state radiation process $e^{+} e^{-} \rightarrow J / \psi \gamma \rightarrow \mu^{+} \mu^{-} \gamma$ and $B_{\mu \mu}(J / \psi)$ branching fraction [6]. The best accuracy of the $\Gamma_{e e} \cdot \mathcal{B}_{\text {hadrons }}$ value has been reached by combining the result on $\Gamma_{e e}[6]$ with $\mathcal{B}_{\text {hadrons }}$ from BES [9].

This work continues a series of experiments on measuring properties of charmonium resonances performed by the KEDR collaboration [12, 13, 15-17]. In 2010 partial widths $\Gamma_{e e} \cdot \mathcal{B}_{e e}(J / \psi)$ and $\Gamma_{e e} \cdot \mathcal{B}_{\mu \mu}(J / \psi)$ were measured with high accuracy of $2.4 \%$ and $2.5 \%$, respectively [12]. In this article we present new results on $\Gamma_{e e}$ and $\Gamma_{e e} \cdot \mathcal{B}_{\text {hadrons }}$ obtained by measuring the cross sections of $e^{+} e^{-} \rightarrow$ hadrons and $e^{+} e^{-} \rightarrow e^{+} e^{-}$as a function of the centre-of-mass (c.m.) energy in the vicinity of the $J / \psi$ resonance with the KEDR detector at the VEPP-4M $e^{+} e^{-}$collider.

\section{VEPP-4M collider and KEDR detector}

A detailed description of the VEPP-4M $e^{+} e^{-}$collider and the KEDR detector (figure 1) can be found in refs. [18, 19].

VEPP-4M $e^{+} e^{-}$collider was designed to operate in the centre of mass energy from 2 to $11 \mathrm{GeV}$. The peak luminosity of the collider in the $J / \psi$ region is about $2 \times 10^{30} \mathrm{~cm}^{-2} \mathrm{~s}^{-1}$ in the $2 \times 2$ bunch operating mode at a beam current of $1.5 \mathrm{~mA}$.

One of the main features of the VEPP-4M is a possibility of precise energy determination. The resonant depolarization method (RDM) [20,21] provided in special calibration 

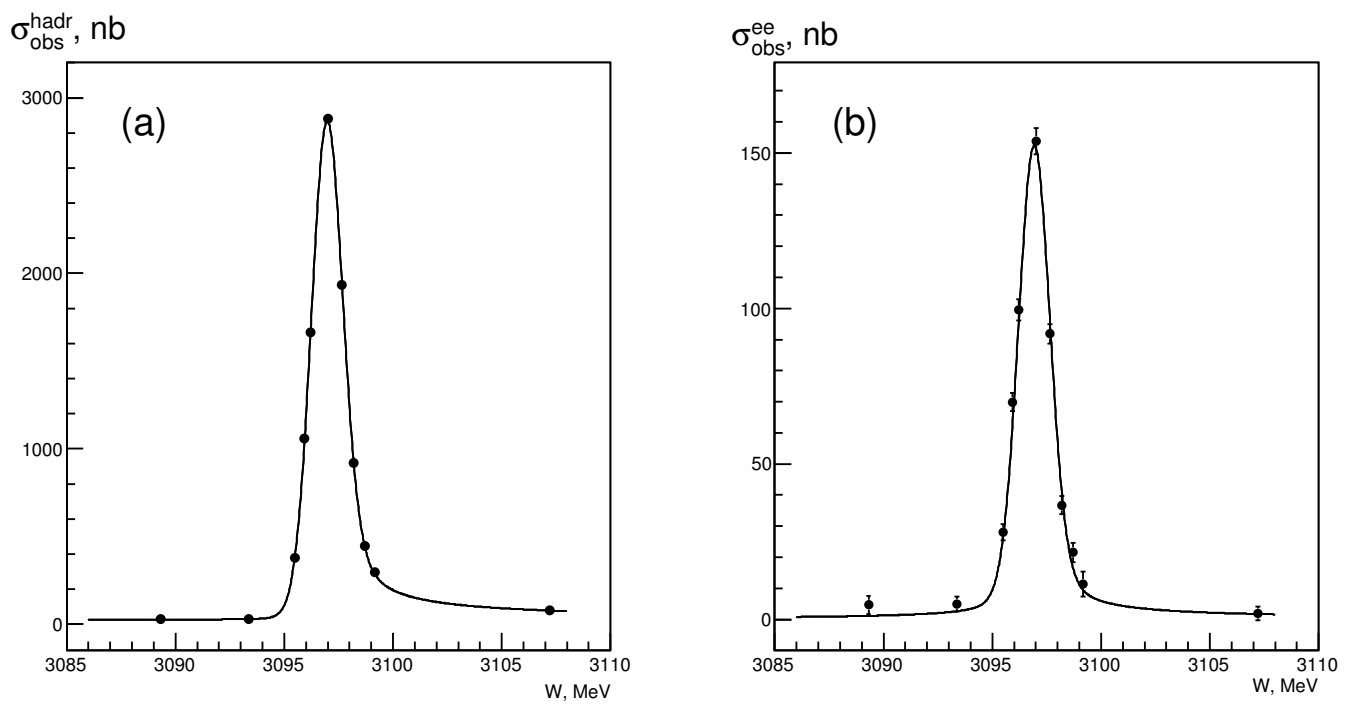

Figure 2. Observed cross section as a function of the c.m. energy (a) for $e^{+} e^{-} \rightarrow J / \psi \rightarrow$ hadrons and (b) for $e^{+} e^{-} \rightarrow J / \psi \rightarrow e^{+} e^{-}$processes. The curves are the result of the combined fit, $\chi^{2}$ of the fit equals 6.6 and 9.0 for the hadronic and leptonic channels, respectively, with the total number of degrees of freedom 15.

runs has the relative accuracy of about $10^{-6}$. Accuracy of energy interpolation to the data taking periods is about to $10-30 \mathrm{keV}[15,17]$. Infrared light Compton backscattering method is used to carry out continuous energy monitoring with the approximate accuracy less than $100 \mathrm{keV}$.

The KEDR detector covers solid angle $95 \%$ of $4 \pi$ and includes the following main components: the vertex detector (VD), the drift chamber (DC), the scintillation time-offlight counters, the aerogel threshold Cherenkov counters, the electromagnetic calorimeter made of liquid krypton (LKr) barrel part and CsI crystals in the end-caps, the muon system inside the magnet yoke. Super-conducting solenoid provide longitudinal magnetic field up to $0.7 \mathrm{~T}$. The detector also contains scattered electron tagging system for studies of twophoton processes. The on-line luminosity measurement is provided by two independent single bremsstrahlung monitors.

\section{Experiment and data sample}

Our analysis is based on the same data set, with an integrated luminosity of $230 \mathrm{nb}^{-1}$, as that used in the KEDR analysis of the leptonic channels [12]. During the scan the data were collected at 11 energy points as shown in figure 2 that allows a fit of the resonance shape and determination of the nonresonant background contributions to be performed. The full data sample corresponds to 250 thousands of produced $J / \psi$ mesons. The beam energy was measured by the resonant depolarization method [20]. 26 calibrations were carried out during the scan, before and after data taking at each energy point. Between the calibrations the beam energy was interpolated with the accuracy better than $15 \mathrm{keV}$. 


\section{$4 e^{+} e^{-}$cross section in vicinity of a narrow resonance}

The cross section for the annihilation process $e^{+} e^{-} \rightarrow$ hadrons in the vicinity of a narrow resonance can be presented in the form [16]:

$$
\begin{aligned}
\sigma_{\text {n.r. }}^{\text {hadr }}(W)= & \frac{12 \pi}{W^{2}}\left\{\left(1+\delta_{\mathrm{sf}}\right)\left[\frac{\Gamma_{e e} \tilde{\Gamma}_{\mathrm{h}}}{\Gamma M} \operatorname{Im} f(W)-\frac{2 \alpha \sqrt{R \Gamma_{e e} \tilde{\Gamma}_{\mathrm{h}}}}{3 W} \lambda \operatorname{Re} \frac{f^{*}(W)}{1-\Pi_{0}}\right]\right. \\
& \left.-\frac{\beta \Gamma_{e e} \tilde{\Gamma}_{\mathrm{h}}}{2 \Gamma M}\left[\left(1+\frac{M^{2}}{W^{2}}\right) \arctan \frac{\Gamma W^{2}}{M\left(M^{2}-W^{2}+\Gamma^{2}\right)}-\frac{\Gamma M}{2 W^{2}} \ln \frac{\left(\frac{M^{2}}{W^{2}}\right)^{2}+\left(\frac{\Gamma M}{W^{2}}\right)^{2}}{\left(1-\frac{M^{2}}{W^{2}}\right)^{2}+\left(\frac{\Gamma M}{W^{2}}\right)^{2}}\right]\right\},
\end{aligned}
$$

where $W$ is the c.m. energy, $M$ is the mass of the resonance, $\Gamma$ is its total width, $\alpha$ is the fine structure constant and $R$ is the ratio $\sigma\left(e^{+} e^{-} \rightarrow\right.$ hadrons $) / \sigma\left(e^{+} e^{-} \rightarrow \mu^{+} \mu^{-}\right)$outside of the resonance region. The truncated vacuum-polarization operator $\Pi_{0}$ does not include a contribution of the resonance itself.

The radiative correction $\delta_{\mathrm{sf}}$ can be obtained from the structure-function approach of ref. [22]:

$$
\begin{aligned}
\delta_{\mathrm{sf}} & =\frac{3}{4} \beta+\frac{\alpha}{\pi}\left(\frac{\pi^{2}}{3}-\frac{1}{2}\right)+\beta^{2}\left(\frac{37}{96}-\frac{\pi^{2}}{12}-\frac{1}{36} \ln \frac{W}{m_{\mathrm{e}}}\right), \\
\beta & =\frac{4 \alpha}{\pi}\left(\ln \frac{W}{m_{\mathrm{e}}}-\frac{1}{2}\right),
\end{aligned}
$$

where $m_{\mathrm{e}}$ is the electron mass. The function $f$ is defined as

$$
f(W)=\frac{\pi \beta}{\sin \pi \beta}\left(\frac{W^{2}}{M^{2}-W^{2}-i M \Gamma}\right)^{1-\beta} .
$$

The parameter $\lambda$ in eq. (4.1) characterizes the strength of the interference effect in the inclusive hadronic cross section. According to ref. [16] the expression for $\lambda$ can be written as

$$
\lambda=\sqrt{\frac{R \mathcal{B}_{e e}}{\mathcal{B}_{\text {hadrons }}}}+\sqrt{\frac{1}{\mathcal{B}_{\text {hadrons }}}} \sum_{m} \sqrt{b_{m} \mathcal{B}_{m}^{(s)}}\left\langle\cos \phi_{m}\right\rangle_{\Theta} .
$$

The summation is performed over all exclusive hadronic modes.

Here and below $\left\langle\cos \phi_{m}\right\rangle_{\Theta}$ and $\left\langle\sin \phi_{m}\right\rangle_{\Theta}$ are the cosine and sine of the relative phase of the strong and electromagnetic amplitudes for the mode $m$ averaged over the phase space of the products, $b_{m}=R_{m} / R$ is the branching fraction of the corresponding continuum process, $\mathcal{B}_{e e}$ is a probability of the decay to an $e^{+} e^{-}$pair, $\mathcal{B}_{\text {hadrons }}$ is the total decay probability to hadrons and $\mathcal{B}_{m}^{(s)}=\Gamma_{m}^{(s)} / \Gamma$, where $\Gamma^{(s)}$ is the contribution of the strong interaction to the partial width for the mode $m$. 
Due to the resonance-continuum interference the effective hadronic width $\tilde{\Gamma}_{\mathrm{h}}$ can differ from the true hadronic partial width $\Gamma_{\text {hadrons }}=\sum_{m} \Gamma_{m}$ :

$$
\tilde{\Gamma}_{\mathrm{h}}=\Gamma_{\text {hadrons }} \times\left(1+\frac{2 \alpha}{3\left(1-\operatorname{Re} \Pi_{0}\right) \mathcal{B}_{\text {hadrons }}} \sqrt{\frac{R}{\mathcal{B}_{e e}}} \times \sum_{m} \sqrt{b_{m} \mathcal{B}_{m}^{(s)}}\left\langle\sin \phi_{m}\right\rangle_{\Theta}\right) .
$$

In this analysis it was assumed that the relative phases of the strong and electromagnetic amplitudes in different decay modes are not correlated. Consequences and experimental verification of this assumption are discussed in detail in refs. [16, 17].

The differential $e^{+} e^{-}$cross section is calculated with

$$
\begin{aligned}
\left(\frac{d \sigma}{d \Omega}\right)^{e e \rightarrow e e}= & \left(\frac{d \sigma}{d \Omega}\right)_{\mathrm{QED}}^{e e \rightarrow e e}+\frac{1}{W^{2}}\left(1+\delta_{s f}\right)\left\{\frac{9}{4} \frac{\Gamma_{e e}^{2}}{\Gamma M}\left(1+\cos ^{2} \theta\right) \operatorname{Im} f\right. \\
& \left.-\frac{3 \alpha}{2} \frac{\Gamma_{e e}}{M}\left[\left(1+\cos ^{2} \theta\right) \operatorname{Re} \frac{f^{*}}{1-\Pi_{0}(s)}-\frac{(1+\cos \theta)^{2}}{(1-\cos \theta)} \operatorname{Re} \frac{f^{*}}{1-\Pi_{0}(t)}\right]\right\},
\end{aligned}
$$

where $s=W^{2}$ and $t=-W^{2} \cdot(1-\cos \theta) / 2$ are the c.m. energy squared and momentum transfer squared, $\theta$ is the electron scattering angle. The first term in eq. (4.7) represents the QED cross section obtained with the Monte Carlo technique [23, 24]. The second term is responsible for the resonance contribution and the third one for the interference. The accuracy of the formulae (4.7) about $0.1 \%$ is sufficient for this work and is confirmed with more precise expressions given in [25].

The detailed description of the extracting $\Gamma_{e e}(J / \psi) \cdot \mathcal{B}_{\text {hadrons }}(J / \psi), \Gamma_{e e}(J / \psi) \cdot \mathcal{B}_{e e}(J / \psi)$ and $\Gamma_{e e}(J / \psi)$ values is given in section 5.5.

\section{Data analysis}

\subsection{MC simulation}

We used MC samples of $J / \psi$ inclusive decays and the continuum multihadron events to obtain the detector efficiency. The samples were generated with the tuned version of the BES generator [26] based on JETSET 7.4 [27]. The procedure of the parameter tuning is discussed in detail in section 6.2. The generated events were reweighted to ensure that the branching fractions of the most probable decay modes correspond to the results of the PDG fit [6]. MC samples of Bhabha events required for the luminosity determination were simulated using the BHWIDE [23] and MCGPJ [24] generators. Generated MC events were then processed with the detector simulation package based on GEANT, version 3.21 [28], and reconstructed with the same conditions as experimental data.

During the data taking in 2005 there was an additional online condition - the number of hits in the VD should not exceed 60 which corresponded to 10 charged tracks. Due to substantial crosstalk in VD electronics, there was some loss of signal events. The effect of crosstalk was carefully simulated.

To take into account the signal and background coincidences, a trigger from arbitrary beam crossings was implemented. The events recorded with this "random trigger" were superimposed with simulated events. 


\section{$5.2 \quad$ Trigger requirements}

The trigger consists of two hardware levels: the primary trigger (PT) and the secondary trigger (ST) [29]. The primary trigger required signals from two or more non-adjacent scintillation counters or an energy deposition in the endcap calorimeter of at least $100 \mathrm{MeV}$. A veto from CsI calorimeter crystals closest to the beam line was used to suppress the machine background. The conditions of the secondary trigger were rather complicated, and were satisfied by events with two tracks in the vertex detector and the drift chamber or with a single track which deposited more the $70 \mathrm{MeV}$ in the barrel calorimeter.

During the offline analysis all events (both recorded in experiment and simulated) were required to pass through the software event filter. It used a digitized response from detector subsystems and applied tighter conditions on its input in order to decrease the effect of calorimeter energy threshold and possible hardware-trigger instability.

\subsection{Luminosity determination}

For the absolute luminosity determination, $e^{+} e^{-}$events in the barrel LKr calorimeter [19] were used taking into account the contribution of $J / \psi$ decays into $e^{+} e^{-}$(see eq. (4.7)).

The final-state radiation (FSR) effects are considered using the PHOTOS package [30]. The $J / \psi \rightarrow e^{+} e^{-}$cross section is shown in figure $2 \mathrm{~b}$ obtained by subtracting the contribution of Bhabha events from the total $e^{+} e^{-} \rightarrow e^{+} e^{-}$cross section.

The $e^{+} e^{-}$event selection includes the following criteria in addition to trigger requirements:

- two clusters within the polar angle range $40<\theta<140^{\circ}$ and the energy $E_{1,2}$ larger than $700 \mathrm{MeV}$ each;

- the energy deposition outside of those two clusters smaller than $10 \%$ of the total energy deposited in the calorimeter $E_{\text {cal }}$;

- acollinearities of the polar $\Delta \theta$ and azimuthal $\Delta \varphi$ angles smaller than $15^{\circ}$;

- event sphericity $S_{\text {ch }}$ calculated with charged particles smaller than 0.05 ;

- two or three tracks in the drift chamber coming from the interaction point: the impact parameter with respect to the beam axis $\rho<0.5 \mathrm{~cm}$, the coordinate of the point of closest approach $\left|\mathrm{z}_{0}\right|<13 \mathrm{~cm}$ and the transverse momentum $P_{\mathrm{t}}>100 \mathrm{MeV}$.

Cosmic background was additionally suppressed with the muon system by veto signals from opposite or adjacent to opposite octants or more than three layers fired in one octant. Alternatively, cosmic events were suppressed with the time-of-flight condition.

Figure 3 shows comparison between $e^{+} e^{-} \rightarrow e^{+} e^{-}$data and MC simulation. The distribution in the electron scattering angle for selected $e^{+} e^{-}$events is shown in figure 4 . The angular distributions of events from Bhabha scattering and from $J / \psi$ decay are different which allows us to separate those contributions at each data point. 

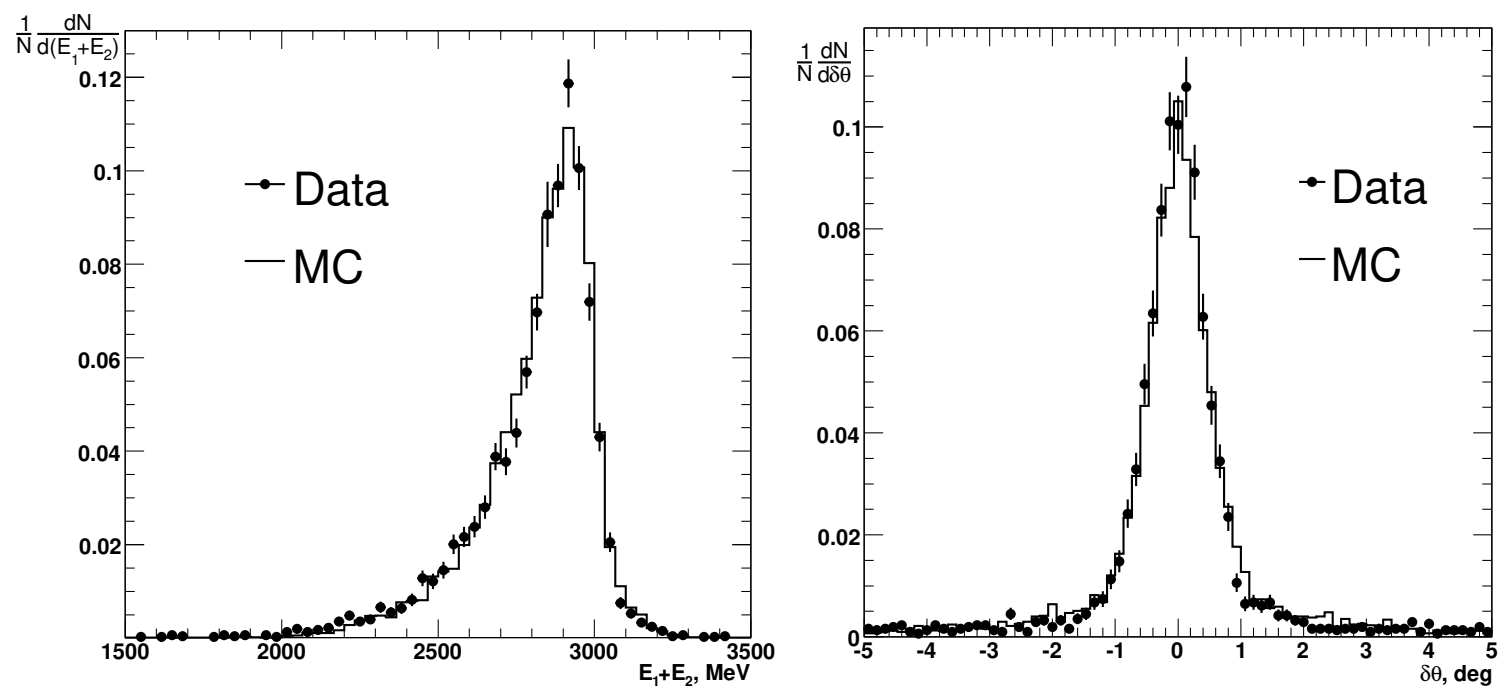

Figure 3. Properties of $e^{+} e^{-}$events produced at the $J / \psi$ peak at the c.m. energy $3097 \mathrm{MeV}$. Sum of two maximum cluster energies (left) and polar-angle acollinearity in degrees (right). All distributions are normalized to unity.

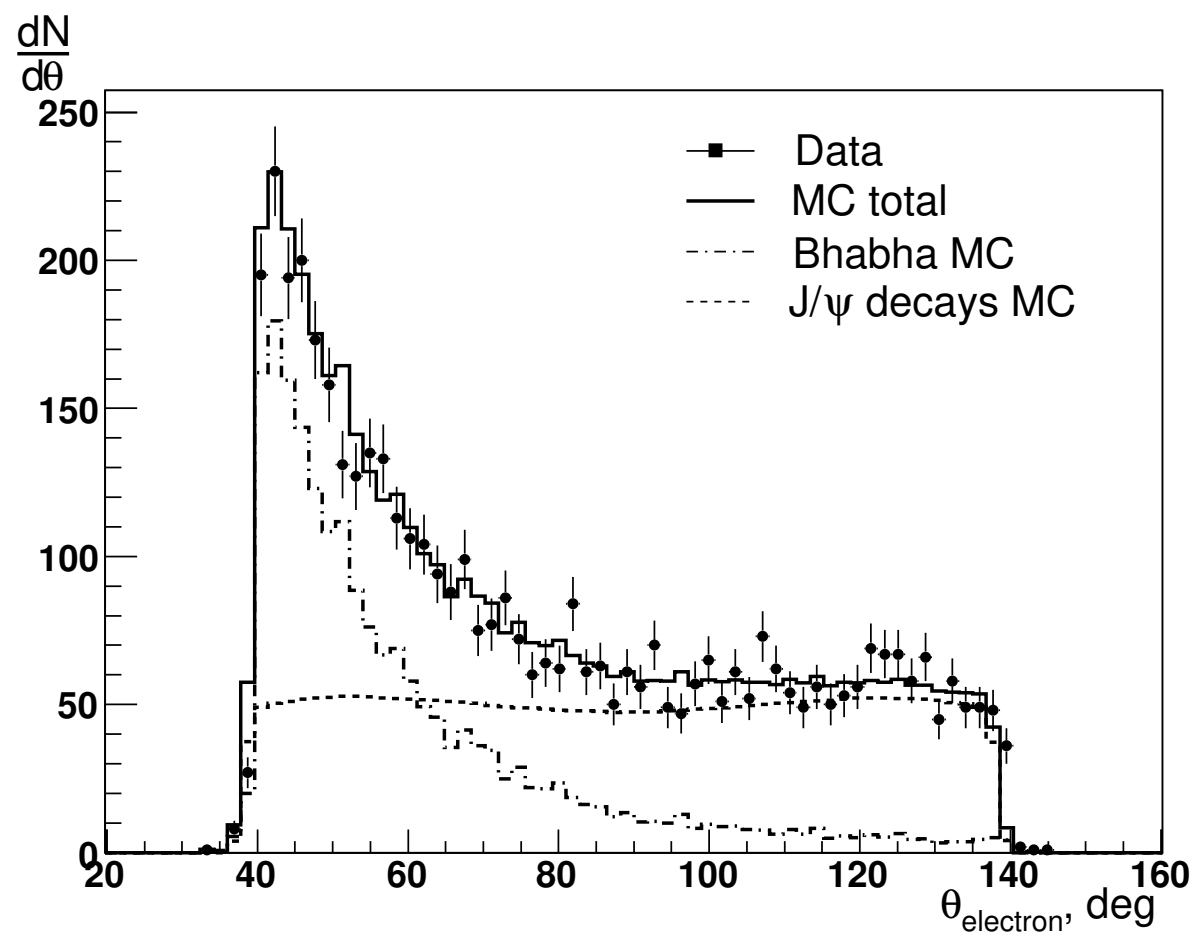

Figure 4. Distribution of the electron polar angle at the energy $3097 \mathrm{MeV}$. The points show experimental data. The histograms correspond to MC simulation: the dashed-doted histogram represents Bhabha scattering, the dashed histogram represents a contribution of the $J / \psi$ resonance and their interference calculated according to eq. (4.7), and the solid-line histogram is the sum of the contributions. 


\subsection{Selection of hadronic events}

In our analysis the following selection requirements are applied:

- total energy deposition in the calorimeter $700<E_{\text {cal }}<2500 \mathrm{MeV}$;

- more than $15 \%$ of total energy deposited in the barel LKr calorimeter $E_{\mathrm{LKr}} / E_{\text {cal }}>0.15$;

- at least one track with $\rho<0.5 \mathrm{~cm},\left|\mathrm{z}_{0}\right|<13 \mathrm{~cm}$ and $P_{\mathrm{t}}>100 \mathrm{MeV}$;

- at least three particles in the detector, including tracks in the drift chamber and calorimeter clusters, which are not associated with any track;

- the ratio of the Fox-Wolfram moments [31] $H_{2} / H_{0}<0.9$.

The requirements on energy deposition separate hadronic events from backgrounds: the upper requirement reduces a fraction of $e^{+} e^{-}$events and the lower one suppresses $\mu^{+} \mu^{-}$and machine backgrounds. The requirement on the ratio of the Fox-Wolfram moments $H_{2} / H_{0}$ is significant in reducing background from quasi-collinear $e^{+} e^{-}$events with additional particles from radiation and interaction with detector material. Cosmic events were additionally suppressed as in selection of $e^{+} e^{-}$events.

Figure 5 shows comparison between the most important event characteristics obtained in the experiment and in the simulation.

\subsection{Fitting of the data}

We performed a combined fit of the data on hadron and $e^{+} e^{-}$production in the energy range of the $J / \psi$ resonance.

Experimental runs were grouped into points according to run energy. The collision energy at each point was determined by interpolating the beam energy measurements and assuming the $e^{+} e^{-}$beam energy symmetry $W=2 E_{\text {beam. }}$. A sample of $e^{+} e^{-}$events was subdivided into 10 equal angular intervals in the range from $40^{\circ}$ to $140^{\circ}$.

The numbers of hadronic $N_{i}$ and leptonic $n_{i j}$ events observed at each energy point and each angular interval were fitted simultaneously as a function of collision energy and electron scattering angle using a minimizing function

$$
\chi^{2}=\sum_{i} \frac{\left(N_{i}^{\exp }-N_{i}^{\text {theor }}\right)^{2}}{N_{i}^{\exp }}+\sum_{i} \sum_{j} \frac{\left(n_{i j}^{\exp }-n_{i j}^{\text {theor }}\right)^{2}}{n_{i j}^{\exp }},
$$

where $N_{i}^{\exp / \text { theor }}$ and $n_{i j}^{\exp / \text { theor }}$ are experimentally measured and theoretically calculated numbers of hadronic and Bhabha events, respectively. Theoretically calculated event numbers were obtained as follows:

$$
\begin{aligned}
N_{i}^{\text {theor }} & =L_{i} \cdot \sigma^{\text {hadr }}\left(W_{i}\right), \\
n_{i j}^{\text {theor }} & =L_{i} \cdot \sigma^{e e}\left(W_{i}, \theta_{j}\right) .
\end{aligned}
$$



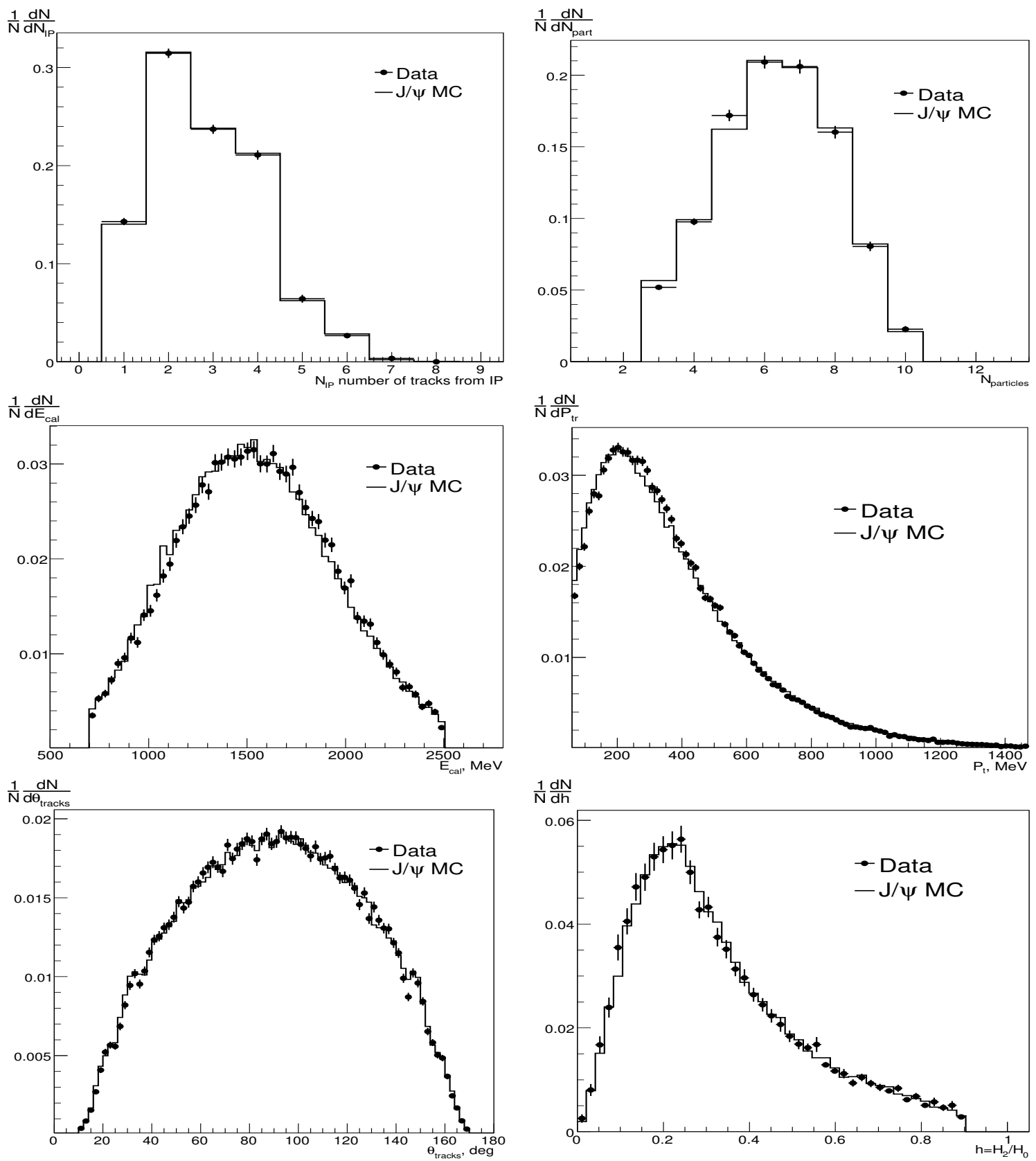

Figure 5. Properties of hadronic events produced in the vicinity of the $J / \psi$ resonance: the number of tracks from the interaction point $N_{\mathrm{IP}}$, the total number of particles $N_{\text {part }}$, energy deposited in the calorimeter $E_{\text {cal }}$, inclusive $P_{\mathrm{t}}$ and $\theta_{\text {tracks }}$ distributions and the ratio of Fox-Wolfram moments $H_{2} / H_{0}$. The points represent experimental data, the histograms correspond to simulation of $J / \psi$ decays. All distributions are normalized to unity. 
Observed cross sections $\sigma^{\text {hadr }}\left(W_{i}\right)$ and $\sigma^{e e}\left(W_{i}, \theta_{j}\right)$ are determined from eq. (4.1) and eq. (4.7), respectively:

$$
\begin{aligned}
\sigma^{\text {hadr }}(W) & =\varepsilon_{\text {hadr }} \int \sigma_{\text {n.r. }}^{\text {hadr }}\left(W^{\prime}\right) G\left(W, W^{\prime}\right) d W^{\prime}+\sigma_{\text {cont }}(W), \\
\sigma^{e e}(W, \theta) & =\varepsilon_{e e}(\theta)\left(\frac{d \sigma}{d \theta}\right)^{e e \rightarrow e e}(W),
\end{aligned}
$$

where the cross section of the annihilation process near the $J / \psi$ resonance is convolved with the Gaussian distribution with the energy spread $\sigma_{W}$ :

$$
G\left(W, W^{\prime}\right)=\frac{g\left(W-W^{\prime}\right)}{\sqrt{2 \pi} \sigma_{W}} e^{-\frac{\left(W-W^{\prime}\right)^{2}}{2 \sigma_{W}^{2}}}
$$

The pre-exponential factor $g$ differs from unity due to some accelerator-related effects. Its impact on the results of the measurements is considered in section 5.4. The continuum cross section is almost constant in the vicinity of a narrow resonance and can be parametrised with

$$
\sigma_{\text {cont }}(W)=\sigma_{0} \cdot\left(\frac{m_{J / \psi}}{W}\right)^{2} .
$$

In eqs. (5.3) and (5.4), $\varepsilon_{\text {hadr }}$ and $\varepsilon_{e e}(\theta)$ are detection efficiencies and their dependence on beam energy can be neglected.

Luminosity $L_{i}$ at $i$-th energy point was determined as:

$$
L_{i}=R_{\mathrm{L}} \cdot L\left(E_{i}\right),
$$

where $L\left(E_{i}\right)$ is the integrated luminosity measured by the bremsstrahlung luminosity monitor at the $i$-th energy point and $R_{\mathrm{L}}$ is an absolute luminosity calibration factor.

The statistical uncertainties of parameters $\Gamma_{e e}(J / \psi), \Gamma_{e e}(J / \psi) \cdot \mathcal{B}_{e e}(J / \psi), \Gamma_{e e}(J / \psi) \cdot$ $\mathcal{B}_{\text {hadrons }}(J / \psi)$ are strongly correlated. To determine these uncertainties accurately, the fit was performed with two sets of free parameters. In the first set the parameters $\Gamma_{e e}(J / \psi)$ and $\Gamma_{e e}(J / \psi) \cdot \mathcal{B}_{e e}(J / \psi)$ were floating. In the second set the parameters $\Gamma_{e e}(J / \psi) \cdot \mathcal{B}_{\text {hadrons }}(J / \psi)$ and $\Gamma_{e e}(J / \psi) \cdot \mathcal{B}_{e e}(J / \psi)$ were floating. Both sets contained auxiliary free parameters: absolute luminosity calibration factor $R_{\mathrm{L}}$, resonance mass $m(J / \psi)$, beam energy spread $\sigma_{W}$ and continuum contribution $\sigma_{0}$. To relate the values of $\Gamma_{e e}(J / \psi), \Gamma_{e e}(J / \psi) \cdot \mathcal{B}_{e e}(J / \psi)$ and $\Gamma_{e e}(J / \psi) \cdot \mathcal{B}_{\text {hadrons }}(J / \psi)$ the ratio $\Gamma_{e^{+} e^{-}} / \Gamma_{\mu^{+} \mu^{-}}(J / \psi)=1.0022 \pm 0.0065$ was fixed from the KEDR result [13] and the variation of the ratio inside its uncertainties introduces negligible systematic uncertainty to the measured values. The results obtained from the fits are listed in table 1 . The $J / \psi$ mass value is in good agreement with that published earlier by the KEDR collaboration [17].

\section{$6 \quad$ Study of systematic uncertainties}

Main contributions of systematic uncertainties to the $\Gamma_{e e}(J / \psi), \Gamma_{e e}(J / \psi) \cdot \mathcal{B}_{\text {hadrons }}(J / \psi)$ and $\Gamma_{e e}(J / \psi) \cdot \mathcal{B}_{e e}(J / \psi)$ values discussed in detail in this section were merged into five categories: absolute luminosity measurement, hadron decay simulation, detector and accelerator effects, theoretical uncertainties. 


\begin{tabular}{|l|l|l|}
\hline Free parameter & Fit 1 & Fit 2 \\
\hline$\Gamma_{e e}(\mathrm{keV})$ & $5.550 \pm 0.056$ & - \\
$\Gamma_{e e} \cdot \mathcal{B}_{\text {hadrons }}(\mathrm{keV})$ & - & $4.884 \pm 0.048$ \\
$\Gamma_{e e} \cdot \mathcal{B}_{e e}(\mathrm{keV})$ & $0.3331 \pm 0.0066$ & $0.3331 \pm 0.0066$ \\
$m(\mathrm{MeV})$ & $3096.902 \pm 0.004$ & $3096.902 \pm 0.004$ \\
$R_{\mathrm{L}}$ & $0.973 \pm 0.008$ & $0.973 \pm 0.008$ \\
$\sigma_{W}(\mathrm{MeV})$ & $0.692 \pm 0.004$ & $0.692 \pm 0.004$ \\
$\sigma_{0}(\mathrm{nb})$ & $28.70 \pm 1.48$ & $28.70 \pm 1.48$ \\
\hline
\end{tabular}

Table 1. Results of two different data fits performed for $\Gamma_{e e}(J / \psi)$ and $\Gamma_{e e}(J / \psi) \cdot \mathcal{B}_{\text {hadrons }}(J / \psi)$ determination.

\begin{tabular}{|l|c|}
\hline Source & Uncertainty, \% \\
\hline Calorimeter alignment & 0.2 \\
Calorimeter response & 0.3 \\
Detection efficiency $\varepsilon_{e e}(\theta)$ & 0.3 \\
MC statistics & 0.2 \\
Cross section & 0.4 \\
Relative luminosity & 0.1 \\
Criterion variation & 0.8 \\
\hline Total & 1.0 \\
\hline
\end{tabular}

Table 2. Systematic uncertainties of the luminosity determination in $\%$.

\subsection{Luminosity uncertainties}

The major sources of the absolute luminosity determination uncertainties are presented in table 2 .

The LKr calorimeter was aligned to the drift chamber using DC-reconstructed tracks from cosmic events. The position of the interaction point and the beam-line direction in the coordinate system of the detector were found using the primary-vertex distribution of hadronic events.

The luminosity uncertainty due to inaccuracy of the alignment was evaluated by applying the one-sigma shift during the reconstruction. The obtained uncertainty is less than $0.2 \%$. The uncertainty due to the imperfect simulation of the calorimeter response was estimated by varying sensitivity to the energy loss fluctuations between LKr calorimeter electrodes and appears to be less than $0.3 \%$.

The detection efficiency function for electrons, $\varepsilon_{e e}(\theta)$, was calculated with $J / \psi \rightarrow e^{+} e^{-}$ simulation, with the $\theta$ angle measured in the drift chamber or LKr calorimeter, the result difference does not exceed $0.3 \%$. A MC statistical uncertainty corresponds to $0.15 \%$. To estimate the uncertainty of the $e^{+} e^{-} \rightarrow e^{+} e^{-}$scattering cross section, calculated from eq. (4.7) two event generators - BHWIDE and MCGPJ were used. The difference in the $\Gamma_{e e}(J / \psi)$ value was $0.37 \%$.

The luminosity spread was estimated as a difference of the results from two independent luminosity monitors and was about $0.4 \%$. This effect was studied with toy MC and the 


\begin{tabular}{|l|l|c|}
\hline Variable & Range variation & Uncertainty, $\%$ \\
\hline$\theta$ & $\theta>40 \div 50^{\circ}$ and $\theta<90 \div 140^{\circ}$ & 0.5 \\
$\left(E_{1}+E_{2}\right)$ & $>1800 \div 2000 \mathrm{MeV}$ & 0.3 \\
$N_{\text {IP definition }}$ & $\rho<0.5 \div 1.0 \mathrm{~cm}$, & 0.3 \\
& $\left|\mathrm{z}_{0}\right|<13 \div 55 \mathrm{~cm}$ and & \\
& $P_{t}>0 \div 100 \mathrm{MeV}$ & \\
$E_{\text {cal }}-\left(E_{1}+E_{2}\right)$ & $<0.1 \div 1.0 E_{\text {cal }}$ & 0.3 \\
$\Delta \theta$ acollinearity & $<15 \div 30^{\circ}$ & 0.2 \\
$\Delta \phi$ acollinearity & $<15 \div 30^{\circ}$ & 0.2 \\
$S_{\text {ch }}$ & $<0.05 \div 0.1$ & 0.2 \\
\hline Total & 0.8 \\
\hline
\end{tabular}

Table 3. Uncertainties in $\%$ due to variation of the selection criteria for $e^{+} e^{-}$events.

corresponding $\Gamma_{e e}(J / \psi)$ and $\Gamma_{e e}(J / \psi) \cdot \mathcal{B}_{\text {hadrons }}(J / \psi)$ width uncertainties were about $0.04 \%$ and $\Gamma_{e e}(J / \psi) \cdot \mathcal{B}_{e e}(J / \psi)$ uncertainty was about $0.06 \%$.

In addition, systematic effects related to luminosity were evaluated by using variation of the selection requirements. The requirement on the polar angle $\theta$ was varied in the broad range, and the corresponding change in the number of selected Bhabha events reached $50 \%$. All variations are summarized in table 3. Those effects can originate from already considered sources and statistical fluctuations. Despite of this we included them in the total uncertainty to get conservative error estimation.

\subsection{Uncertainty due to imperfect simulation of $J / \psi$ decays}

The next important source of uncertainties on the $\Gamma_{e e}(J / \psi)$ value is the imperfect simulation of $J / \psi$ decays. To tune the simulation procedure and obtain a reliable estimate of the systematic uncertainty, we follow the method used in ref. [16].

Let us discuss the idea of the method in brief. Assume that we have a perfect simulation procedure capable of reproducing all event characteristics and correlations between them, but it has a set of internal parameters to be tuned. By varying one of the parameters, one should trace the change of the mean value of some observable, for example the mean multiplicity $\left\langle N_{\mathrm{IP}}\right\rangle$, and the detection efficiency $\varepsilon$. The simulated value of the observable coincides with the measured one at the optimal setting of the parameter. For small variations the detection efficiency linearly depends on the mean multiplicity, therefore the accuracy of the efficiency determination $\delta \varepsilon=\partial \varepsilon / \partial\left\langle N_{\mathrm{IP}}\right\rangle \delta\left\langle N_{\mathrm{IP}}\right\rangle$, where $\delta\left\langle N_{\mathrm{IP}}\right\rangle$ is the uncertainty of the experimental value of the multiplicity. In case of several simulation parameters to vary, one should get the set of $\varepsilon\left(N_{\mathrm{IP}}\right)$ trajectories crossing together at the point which corresponds to an experimental observable. In practice, the simulation procedure is not perfect, thus instead of one intersection point we have the situation depicted in figure 6 . The uncertainty of the detection efficiency grows due to difference in trajectory slopes obtained with variations of simulation parameters. The estimate of the uncertainty interval corresponds to the vertical size of the shadow box in figure 6 while the horizontal size is determined by the track multiplicity uncertainty in the experiment. 


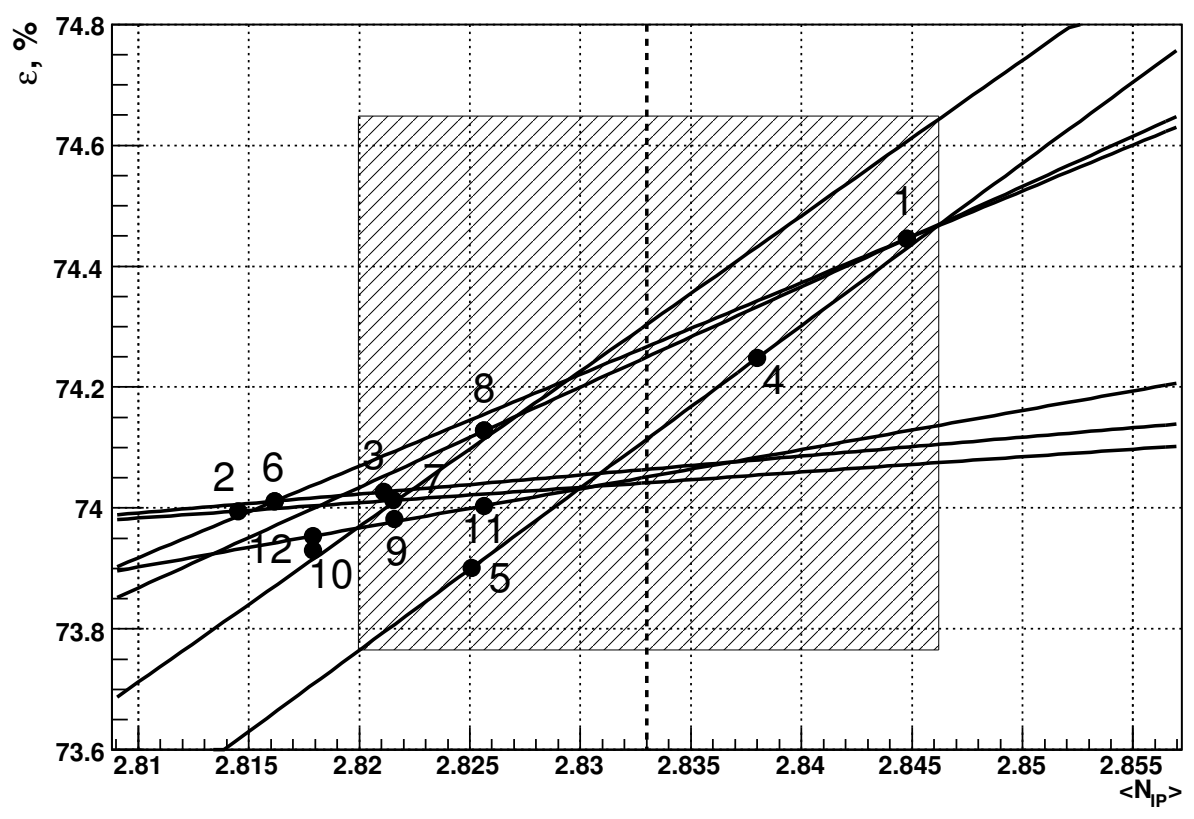

Figure 6. Detection efficiency dependence on charged multiplicity for different versions of the $J / \psi$ decay simulation. The solid lines correspond to variation of the selected parameters. The dotted line corresponds to the experimental measured charged multiplicity and the rectangular shadow box shows its statistical error. The vertical limits of the shadow box correspond to fit lines crossing the data statistical bounds.

To obtain the results presented in figure 6, we iterated as follows: vary one of the JETSET parameters and then modify some complementary parameter to achieve good agreement in observed charged multiplicity. The values of the mean multiplicity and the detection efficiencies obtained for various settings of parameters are summarized in table 4 .

The main JETSET parameters to vary are PARJ(21), PARJ(33), PARJ(37), PARJ(41) and $\operatorname{PAR}(42)$ referred to $\sigma_{\mathrm{P}_{\mathrm{T}}}, W_{\text {stop }}, \delta W_{\text {stop }}$ and two parameters $a$ and $b$ for the Lund fragmentation function, respectively. The parameter $\sigma_{\mathrm{P}_{\mathrm{T}}}$ is responsible for a width in the Gaussian transverse-momentum distributions of primary particles appearing during fragmentation, while $W_{\text {stop }}$ is the energy of the jet system below which a final hadron pair is produced. This energy is smeared with a relative width $\delta W_{\text {stop }}$. Beside variations of fragmentation function parameters, we tried the fragmentation with parton showers switched off.

The charged multiplicity was selected for tuning as the most sensitive event characteristic. In addition to it, simulated distributions of charged tracks sphericity, Fox-Wolfram moments, energy deposited in the calorimeter, the inclusive event characteristics such as momentum, azimuthal and polar angles, were checked for agreement with experimental data. Histogram shapes were compared using a Kolmogorov test and simulated samples that gave the values of the Kolmogorov test lower than 0.6 were rejected.

The multihadron efficiency was averaged over efficiencies corresponding to an experimentally measured charged multiplicity $\left\langle N_{\mathrm{IP}}\right\rangle$ in figure 6 and equals $74.2 \pm 0.4 \%$.

For the calculation of the mean multiplicity some track selection criteria are required. Their choice leads to an additional uncertainty on the detection efficiency which is smaller 


\begin{tabular}{|c|c|c|c|c|c|c|}
\hline Version & \multicolumn{2}{|c|}{ JETSET modifications } & $\left\langle N_{\text {IP }}\right\rangle$ & $\varepsilon, \%$ & $k$-test & $\chi_{\text {MC-data }}^{2} / n d f$ \\
\hline \multicolumn{7}{|c|}{$\sigma_{\mathrm{P}_{\mathrm{T}}}$ and $\delta W_{\text {stop }}$ varied } \\
\hline \multicolumn{7}{|c|}{\begin{tabular}{l|l}
$\sigma_{P_{T}}, \mathrm{GeV}$ & $\delta W_{\text {stop }}$ \\
\end{tabular}} \\
\hline 1 & 0.55 & 0.2 & 2.845 & 74.445 & 0.950 & $5.924 / 7$ \\
\hline 2 & 0.7 & 0.2 & 2.816 & 74.010 & 0.806 & $9.596 / 7$ \\
\hline 3 & 0.7 & 0.17 & 2.821 & 74.027 & 0.933 & $7.282 / 7$ \\
\hline \multicolumn{7}{|c|}{ Switching parton showers } \\
\hline 4 & 0.6 & 0.2 & 2.838 & 74.248 & 0.996 & $4.387 / 7$ \\
\hline $5,{ }^{*}$ & 0.6 & 0.2 & 2.825 & 73.901 & 0.999 & $6.697 / 7$ \\
\hline \multicolumn{7}{|c|}{$W_{\text {stop }}$ varied } \\
\hline & $\sigma_{P_{T}}, \mathrm{GeV}$ & $W_{\text {stop }}, \mathrm{GeV}$ & & & & \\
\hline 6 & 0.65 & 0.56 & 2.815 & 73.995 & 0.663 & $8.497 / 7$ \\
\hline 7 & 0.65 & 0.52 & 2.822 & 74.013 & 0.903 & $4.761 / 7$ \\
\hline \multicolumn{7}{|c|}{ Fragmentation function with $a=0.2, b=0.58$} \\
\hline & $\sigma_{\mathrm{P}_{\mathrm{T}}}, \mathrm{GeV}$ & $\delta W_{\text {stop }}, \mathrm{GeV}$ & & & & \\
\hline 8 & 0.65 & 0.2 & 2.826 & 74.128 & 0.954 & $8.574 / 7$ \\
\hline 9 & 0.65 & 0.17 & 2.822 & 73.982 & 0.839 & $13.288 / 7$ \\
\hline 10 & 0.7 & 0.2 & 2.818 & 73.930 & 0.685 & $11.234 / 7$ \\
\hline \multicolumn{7}{|c|}{ Parameters of fragmentation function varied } \\
\hline & $a$ & $b$ & & & & \\
\hline 11 & 1.0 & 0.7 & 2.826 & 74.004 & 0.979 & $10.483 / 7$ \\
\hline 12 & 0.5 & 0.65 & 2.818 & 73.954 & 0.986 & $9.514 / 7$ \\
\hline
\end{tabular}

${ }^{*}$ Switched-off parton shower.

Table 4. Comparison of different versions of MC simulation for $J / \psi$ decays. JETSET modification parameters are presented. For each simulated sample, the detection efficiency was calculated and results of Kolmogorov and $\chi^{2}$ tests on the charged multiplicity distribution are shown as well as average value.

than $0.3 \%$. The track reconstruction efficiency is not exactly the same for the experimental data and simulation. The difference was studied using Bhabha events and cosmic tracks and the appropriate correction was introduced in the detector simulation with an uncertainty smaller than $0.1 \%$.

For reweighting we used significant and well-measured $J / \psi$ decay branching fractions. To check a systematic uncertainty, the remaining branching fractions were added to the list and corresponding MC event weights were recalculated. This leads to uncertainty of less than $0.1 \%$ on the measured $\Gamma_{e e}(J / \psi)$ and $\Gamma_{e e}(J / \psi) \cdot \mathcal{B}_{\text {hadrons }}(J / \psi)$ values.

All systematic uncertainties due to imperfect simulation are summarized in table 5 . 


\begin{tabular}{|l|c|}
\hline Source & Uncertainty, \% \\
\hline Generator & 0.6 \\
Track selection & 0.3 \\
MC statistics & 0.3 \\
Tracking efficiency & 0.1 \\
PDG branchings & 0.1 \\
\hline Total & 0.7 \\
\hline
\end{tabular}

Table 5. Systematic uncertainties of $\Gamma_{e e}(J / \psi) \cdot \mathcal{B}_{\text {hadrons }}(J / \psi)$ due to imperfect simulation of $J / \psi$ decays.

\begin{tabular}{|l|c|}
\hline Source & Uncertainty, \% \\
\hline Cosmic suppression & 0.3 \\
Nuclear interaction & 0.2 \\
Tracking $P_{\mathrm{t}} / \theta$ resolution & 0.2 \\
Trigger efficiency & 0.5 \\
Criterion variation & 0.5 \\
\hline Total & 0.8 \\
\hline
\end{tabular}

Table 6. Sources of detector-related systematic uncertainties in $\%$.

\subsection{Detector-related uncertainties}

The major sources of the detector-related systematic uncertainties in the $\Gamma_{e e}(J / \psi)$ width are listed in table 6 .

To estimate the uncertainty related to the cosmic background, the condition on the muon system veto was replaced with a condition on the average ToF time with the number of hits in the muon system not larger than two. The difference was found to be about $0.3 \%$ for $\Gamma_{e e}(J / \psi)$ and $\Gamma_{e e}(J / \psi) \cdot \mathcal{B}_{\text {hadrons }}(J / \psi)$ and $0.1 \%$ for $\Gamma_{e e}(J / \psi) \cdot \mathcal{B}_{e e}(J / \psi)$.

In addition, we used two models of nuclear interaction during simulation - with the GHEISHA [32] and FLUKA [33] packages as they are implemented in GEANT 3.21. The variation of the resulting $\Gamma_{e e}$ value was about $0.2 \%$.

The two methods to achieve data and MC agreement in the momentum resolution and the angular resolution were used: we scale either the assumed systematic uncertainties of $\mathrm{x}(\mathrm{t})$ determination or the spatial resolution of the drift chamber. That gives a $0.2 \%$ systematic uncertainty.

The trigger inefficiency includes three contributions. The inefficiency of time-of-flight counters used in the primary trigger was determined with especially selected $e^{+} e^{-} \rightarrow$ $e^{+} e^{-}$and cosmic events and equals $0.3 \%$. A systematic uncertainty due to crosstalk in VD electronics was evaluated as a difference of results with two sets of VD simulation parameters obtained by using cosmic and Bhabha events. It was about $0.2 \%$. And the last effect is a veto from CsI crystals near the beam line, which was estimated by varying corresponding trigger thresholds and equals $0.3 \%$.

The effects of possible sources of the detector-related uncertainties were also evaluated by varying the event selection requirements. Minimum and maximum total energies de- 


\begin{tabular}{|l|l|c|}
\hline Variable & Range variation & Uncertainty, \% \\
\hline$E_{\text {cal }}$ & $E_{\text {cal }}>500 \div 700$ and & 0.3 \\
& $E_{\text {cal }}<2500 \div 2700 \mathrm{MeV}$ & \\
$E_{\mathrm{LKr}} / E_{\text {cal }}$ & $>0 \div 0.15$ & 0.3 \\
$N_{\mathrm{IP}}$ & $\rho<0.5 \div 1.5 \mathrm{~cm}, \mathrm{z}_{0}<13 \div 18 \mathrm{~cm}$, & 0.2 \\
& $P_{\mathrm{t}}>60 \div 100 \mathrm{MeV}, N_{\mathrm{IP}}>1$ & \\
$H_{2} / H_{0}$ & $<0.9 \div 1$ & 0.1 \\
\hline Total & & 0.5 \\
\hline
\end{tabular}

Table 7. $\Gamma_{e e}(J / \psi) \cdot \mathcal{B}_{\text {hadrons }}(J / \psi)$ uncertainty in $\%$ due to variation of the selection criteria for hadronic events.

posited in the calorimeter were varied to 500 and $2700 \mathrm{MeV}$, respectively. A requirement on the Fox-Wolfram moments was removed from selection. A requirement on the number of tracks from interaction points was tightened to have $N_{\text {IP }}>1$ and track selections on $\rho, \mathrm{z}_{0}$ and $P_{\mathrm{t}}$ were varied and the obtained difference did not exceed $0.2 \%$. The results are presented in table 7 giving in total about $0.5 \%$.

\subsection{Accelerator uncertainties}

The influence of the machine background was estimated by using a data set collected with separated beams. The number of hadronic events selected from this data set was rescaled to the full data sample proportionally to the integrals of the beam currents. The contribution of background events to the observed cross section is about $6-12 \mathrm{nb}$. The number of selected hadron events was corrected for the number of estimated background events and the data were refitted. The relative uncertainty does not exceed $0.2 \%$.

The non-Gaussian effects in the total collision energy distribution contribute about $0.2 \%$ to the $\Gamma_{e e}(J / \psi)$ uncertainty. To estimate this contribution, we added a preexponential factor while convolving the cross section with a Gaussian function in eq. (5.5) (details are discussed in [17]):

$$
g(\Delta W)=\left(1+a \cdot \Delta W+b \cdot \Delta W^{2}\right) /\left(1+b \cdot \sigma_{W}^{2}\right) .
$$

To check the uncertainty related to the beam energy determination, the values of energy assigned to the data points were corrected within their errors using the known shape of the resonance cross section. For that, eleven free parameters $E_{i}^{\mathrm{fit}}$ were introduced in the fit function (5.1) and the compensating term,

$$
\chi_{E}^{2}=\sum_{i} \frac{\left(E_{i}^{\mathrm{fit}}-E_{i}\right)^{2}}{\sigma_{\mathrm{E}_{i}}^{2}},
$$

was added, where $E_{i}$ is the energy obtained from interpolation of the resonance depolarization data and $\sigma_{\mathrm{E}_{i}}$ is its estimated accuracy. For the cross section calculations, the values $W_{i}=2 E_{i}^{\text {fit }}$ were used. The variation of the fitted $\Gamma_{e e}$ value was about $0.3 \%$.

The list of accelerator uncertainties is presented in table 8. 


\begin{tabular}{|l|c|}
\hline Source & Uncertainty, \% \\
\hline Collider background & 0.2 \\
Non-Gaussian energy & 0.2 \\
Beam energy & 0.3 \\
\hline Total & 0.4 \\
\hline
\end{tabular}

Table 8. Accelerator-related uncertainties contributions in $\%$.

\subsection{Other uncertainties}

To verify the uncertainty related to the interference parameter $\lambda$ parameter, we left $\lambda$ floating resulting in a shift of $0.2 \%$ on the $\Gamma_{e e}(J / \psi)$ and $\Gamma_{e e}(J / \psi) \cdot \mathcal{B}_{\text {hadrons }}(J / \psi)$ values and about $0.1 \%$ on the $\Gamma_{e e}(J / \psi) \cdot \mathcal{B}_{e e}(J / \psi)$ value.

The interference parameter $\lambda$ in the fit was fixed at the value of 0.39 assuming that the sum in (4.5) vanishes. As a crosscheck deviation of $\tilde{\Gamma}_{\mathrm{h}}$ from a sum of partial hadronic widths $\Gamma_{\text {hadrons }}$ due to interference effects was estimated in the Bayesian approach under the assumption that all phases in eq. (4.5) have equal probability as discussed in [16]. The effect depends on the fitted value and its uncertainty $\lambda=0.36 \pm 0.14$ and does not exceed $0.3 \%$.

The accuracy of the analytic expression (4.1) is about $0.1 \%$. In addition, the $0.1 \%$ accuracy of the radiative-correction calculation [22] should be taken into account. The inaccuracy of simulation of FSR effects with PHOTOS is negligible in our analysis.

The sum in quadrature of all contributions listed in this subsection is about $0.4 \%$.

\section{Summary}

The parameters of the $J / \psi$ meson have been measured by using the data collected with the KEDR detector at the VEPP-4M $e^{+} e^{-}$collider. Two data fits were performed, the first one was used to obtain partial lepton widths $\Gamma_{e e}(J / \psi) \cdot \mathcal{B}_{\text {hadrons }}(J / \psi)$ and $\Gamma_{e e}(J / \psi) \cdot \mathcal{B}_{e e}(J / \psi)$. Their errors are strongly correlated, therefore to obtain the total leptonic width $\Gamma_{e e}(J / \psi)$ a separate fit was introduced. Our results are

$$
\begin{aligned}
\Gamma_{e e}(J / \psi) & =5.550 \pm 0.056 \pm 0.089 \mathrm{keV}, \\
\Gamma_{e e}(J / \psi) \cdot \mathcal{B}_{\text {hadrons }}(J / \psi) & =4.884 \pm 0.048 \pm 0.078 \mathrm{keV}, \\
\Gamma_{e e}(J / \psi) \cdot \mathcal{B}_{e e}(J / \psi) & =0.3331 \pm 0.0066 \pm 0.0040 \mathrm{keV}
\end{aligned}
$$

The first and second uncertainties are statistical and systematic, respectively. The major sources of the systematic uncertainties for the $\Gamma_{e e}(J / \psi)$ and $\Gamma_{e e}(J / \psi) \cdot \mathcal{B}_{\text {hadrons }}(J / \psi)$ values are summarized in table 9 and the total systematic uncertainty equals $1.6 \%$. For the $\Gamma_{e e}(J / \psi) \cdot \mathcal{B}_{e e}(J / \psi)$ product, the total systematic uncertainty equals $1.2 \%$.

Our result for the $\Gamma_{e e}(J / \psi) \cdot \mathcal{B}_{\text {hadrons }}(J / \psi)$ value is consistent with and four times more precise than the previous direct measurement in the hadronic channel [9]. The obtained $\Gamma_{e e}(J / \psi) \cdot \mathcal{B}_{e e}(J / \psi)$ value is in good agreement with our previous measurement [12] and supersedes it. 

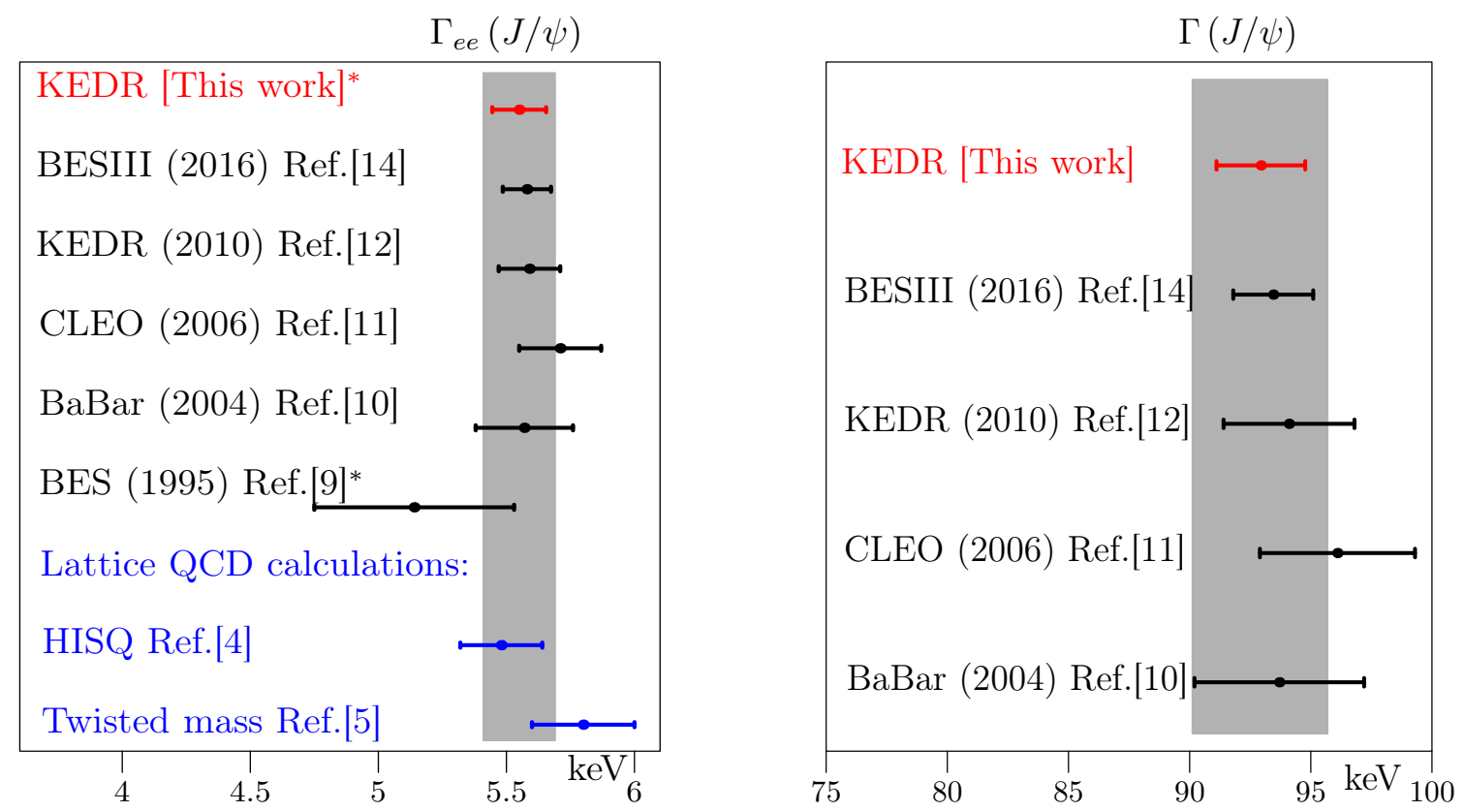

${ }^{*}$ Direct measurement.

Figure 7. Comparison of $\Gamma_{e e}(J / \psi)$ and $\Gamma(J / \psi)$ measured in the most precise experiments and $\Gamma_{e e}(J / \psi)$ predictions from lattice QCD calculations. The $\Gamma(J / \psi)$ value from the BESIII experiment was calculated from [14] using the world-average lepton branching fraction [6]. The gray band corresponds to the world-average value with allowance for the uncertainty in it.

\begin{tabular}{|l|c|c|c|}
\hline \multirow{2}{*}{ Source } & \multicolumn{3}{|c|}{ Uncertainty, \% } \\
\cline { 2 - 4 } & $\Gamma_{e e}$ & $\Gamma_{e e} \cdot \mathcal{B}_{\text {hadrons }}$ & $\Gamma_{e e} \cdot \mathcal{B}_{e e}$ \\
\hline Luminosity & 1.0 & 1.0 & 1.0 \\
Simulation of $J / \psi$ decays & 0.7 & 0.7 & - \\
Detector response & 0.8 & 0.8 & 0.4 \\
Accelerator-related effects & 0.4 & 0.4 & 0.4 \\
Theoretical uncertainties & 0.4 & 0.4 & 0.2 \\
\hline Total & 1.6 & 1.6 & 1.2 \\
\hline
\end{tabular}

Table 9. Dominant systematic uncertainties in the $\Gamma_{e e}(J / \psi), \Gamma_{e e}(J / \psi) \cdot \mathcal{B}_{\text {hadrons }}(J / \psi)$ and $\Gamma_{e e}(J / \psi) \cdot \mathcal{B}_{e e}(J / \psi)$ values.

Taking into account $\mathcal{B}_{e e}(J / \psi)=(5.971 \pm 0.032) \%$ from [6] we determined the total width of the $J / \psi$ meson:

$$
\Gamma=92.94 \pm 1.83 \mathrm{keV} .
$$

The leptonic and total widths of the $J / \psi$ meson $\Gamma_{e e}$ are known from the BESIII [14], CLEO [11] and BaBar [10] experiments. The values were calculated from $\Gamma_{e e}(J / \psi) \cdot \mathcal{B}_{\mu \mu}(J / \psi)$ measured in the radiation process $e^{+} e^{-} \rightarrow \mu^{+} \mu^{-} \gamma$ with the $J / \psi$ meson decaying to muon pair.

The electronic and total widths obtained in our analysis agree well with the world average $\Gamma_{e e}=5.55 \pm 0.14 \pm 0.02 \mathrm{keV}$ and $\Gamma=92.9 \pm 2.8 \mathrm{keV}$ [6]. Figure 7 represents comparison of our $\Gamma_{e e}(J / \psi)$ and $\Gamma(J / \psi)$ results with those obtained in previous experiments. 


\section{Acknowledgments}

We greatly appreciate the efforts of the staff of VEPP-4M to provide good operation of the accelerator complex.

This work was supported by Russian Science Foundation under project N 14-50-00080. Work related to $J / \psi$ Monte Carlo generator tuning was partially supported by Russian Foundation for Basic Research under grant 12-02-01076-a.

Open Access. This article is distributed under the terms of the Creative Commons Attribution License (CC-BY 4.0), which permits any use, distribution and reproduction in any medium, provided the original author(s) and source are credited.

\section{References}

[1] J.H. Kühn, M. Steinhauser and C. Sturm, Heavy Quark Masses from Sum Rules in Four-Loop Approximation, Nucl. Phys. B 778 (2007) 192 [hep-ph/0702103] [INSPIRE].

[2] J.H. Kühn, Precise heavy quark masses, Mod. Phys. Lett. A 28 (2013) 1360019 [INSPIRE].

[3] F. Jegerlehner, Leading-order hadronic contribution to the electron and muon $g-2$, EPJ Web Conf. 118 (2016) 01016 [arXiv: 1511.04473] [INSPIRE].

[4] HPQCD collaboration, G.C. Donald et al., Precision tests of the $J / \psi$ from full lattice QCD: mass, leptonic width and radiative decay rate to $\eta_{c}$, Phys. Rev. D 86 (2012) 094501 [arXiv: 1208.2855] [INSPIRE].

[5] D. Becirevic and F. Sanfilippo, Lattice QCD study of the radiative decays $J / \psi \rightarrow \eta_{c} \gamma$ and $h_{c} \rightarrow \eta_{c} \gamma$, JHEP 01 (2013) 028 [arXiv: 1206.1445] [INSPIRE].

[6] Particle Data Group collaboration, C. Patrignani et al., Review of Particle Physics, Chin. Phys. C 40 (2016) 100001 [InSPIRE].

[7] A.M. Boyarski et al., The Quantum Numbers and Decay Widths of the $\psi(3095)$, Phys. Rev. Lett. 34 (1975) 1357 [INSPIRE].

[8] R. Baldini-Celio et al., Multi-Hadronic Decays and Partial Widths of the $J / \psi(3100)$ Resonance Produced in $e^{+} e^{-}$Annihilation at ADONE, Phys. Lett. B 58 (1975) 471 [INSPIRE].

[9] BES collaboration, J.Z. Bai et al., A Measurement of $J / \psi$ decay widths, Phys. Lett. B 355 (1995) 374 [Erratum ibid. B 363 (1995) 267] [INSPIRE].

[10] BABAR collaboration, B. Aubert et al., $J / \psi$ production via initial state radiation in $e^{+} e^{-} \rightarrow \mu^{+} \mu^{-} \gamma$ at an $e^{+} e^{-}$center-of-mass energy near $10.6 \mathrm{GeV}$, Phys. Rev. D 69 (2004) 011103 [hep-ex/0310027] [INSPIRE].

[11] CLEO collaboration, G.S. Adams et al., Measurement of $\Gamma_{e e}(J / \psi), \Gamma_{\text {tot }}(J / \psi)$, and $\Gamma_{e e}[\psi(2 S)] / \Gamma_{e e}(J / \psi)$, Phys. Rev. D $73(2006) 051103$ [hep-ex/0512046] [INSPIRE].

[12] KEDR collaboration, V.V. Anashin et al., Measurement of $\Gamma_{e e}(J / \psi) \cdot \mathscr{B}\left(J / \psi \rightarrow e^{+} e^{-}\right)$and $\Gamma_{e e}(J / \psi) \cdot \mathscr{B}\left(J / \psi \rightarrow \mu^{+} \mu^{-}\right)$, Phys. Lett. B 685 (2010) 134 [arXiv:0912.1082] [INSPIRE].

[13] KEDR collaboration, V.M. Aulchenko et al., Measurement of the ratio of the leptonic widths $\Gamma_{e e} / \Gamma_{\mu \mu}$ for the $J / \psi$ meson, Phys. Lett. B 731 (2014) 227 [arXiv:1311.5005] [INSPIRE]. 
[14] BESIII collaboration, M. Ablikim et al., Measurement of the leptonic decay width of $J / \psi$ using initial state radiation, Phys. Lett. B 761 (2016) 98 [arXiv:1604.01924] [INSPIRE].

[15] KEDR collaboration, V.M. Aulchenko et al., New precision measurement of the $J / \psi$ and $\psi^{\prime}$ meson masses, Phys. Lett. B 573 (2003) 63 [hep-ex/0306050] [INSPIRE].

[16] KEDR collaboration, V.V. Anashin et al., Measurement of main parameters of the $\psi(2 S)$ resonance, Phys. Lett. B 711 (2012) 280 [arXiv:1109.4215] [INSPIRE].

[17] KEDR collaboration, V.V. Anashin et al., Final analysis of KEDR data on $J / \psi$ and $\psi(2 S)$ masses, Phys. Lett. B 749 (2015) 50 [InSPIRE].

[18] V.V. Anashin et al., VEPP-4M Collider: Status and Plans, in proceedings of the 6th European Particle Accelerator Conference (EPAC 98), Stockholm, Sweden, 22-26 June 1998, S. Myers, L. Liijeby, C. Petit-Jean-Genaz, J. Poole and K.-G. Rensfelt eds., IOP Publishing, Philadelphia U.S.A. (1998), p. 400.

[19] KEDR collaboration, V.V. Anashin et al., The KEDR detector, Phys. Part. Nucl. 44 (2013) 657 [INSPIRE].

[20] A.D. Bukin et al., Absolute calibration of beam energy in the storage ring, $\Phi$-meson mass measurement, Preprint IYF-75-64 (1975) and online pdf version at http://wwwold.inp.nsk.su/activity/preprints/files/1975_064.pdf.

[21] A.N. Skrinsky and Y.M. Shatunov, Precision measurements of masses of elementary particles using storage rings with polarized beams, Sov. Phys. Usp. 32 (1989) 548 [INSPIRE].

[22] E.A. Kuraev and V.S. Fadin, On Radiative Corrections to $e^{+} e^{-}$Single Photon Annihilation at High-Energy, Sov. J. Nucl. Phys. 41 (1985) 466 [InSPIRE].

[23] S. Jadach, W. Placzek and B.F.L. Ward, BHWIDE 1.00: $O(\alpha)$ YFS exponentiated Monte Carlo for Bhabha scattering at wide angles for LEP1/SLC and LEP2, Phys. Lett. B 390 (1997) 298 [hep-ph/9608412] [INSPIRE].

[24] A.B. Arbuzov, G.V. Fedotovich, F.V. Ignatov, E.A. Kuraev and A.L. Sibidanov, Monte-Carlo generator for $e^{+} e^{-}$annihilation into lepton and hadron pairs with precise radiative corrections, Eur. Phys. J. C 46 (2006) 689 [hep-ph/0504233] [INSPIRE].

[25] X.-Y. Zhou, Y.-D. Wang and L.-G. Xia, Analytic forms for cross sections of di-lepton production from $e^{+} e^{-}$collisions around the $J / \psi$ resonance, Chin. Phys. C 41 (2017) 083001 [arXiv: 1701.00218] [INSPIRE].

[26] J.C. Chen, G.S. Huang, X.R. Qi, D.H. Zhang and Y.S. Zhu, Event generator for $J / \psi$ and $\psi(2 S)$ decay, Phys. Rev. D 62 (2000) 034003 [InSPIRE].

[27] T. Sjöstrand, High-energy physics event generation with PYTHIA 5.7 and JETSET 7.4, Comput. Phys. Commun. 82 (1994) 74 [INSPIRE].

[28] R. Brun et al., GEANT Detector Description and Simulation Tool, CERN-W5013 (1994) [INSPIRE].

[29] S.E. Baru et al., Trigger of the KEDR detector, Instrum. Exp. Tech. 54 (2011) 335 [inSPIRE].

[30] E. Barberio and Z. Was, PHOTOS: A Universal Monte Carlo for QED radiative corrections. Version 2.0, Comput. Phys. Commun. 79 (1994) 291 [INSPIRE]. 
[31] G.C. Fox and S. Wolfram, Event Shapes in $e^{+} e^{-}$Annihilation, Nucl. Phys. B 149 (1979) 413 [Erratum ibid. B 157 (1979) 543] [INSPIRE].

[32] H.C. Fesefeldt, The simulation of hadronic showers: physics and applications, PITHA-85-02 (1985) [INSPIRE] and online pdf version at http://cds.cern.ch/record/162911/files/ CM-P00055931.pdf.

[33] A. Fasso et al., The FLUKA code: Present applications and future developments, eConf $\mathbf{C}$ 0303241 (2003) MOMT004 [physics/0306162] [INSPIRE]. 